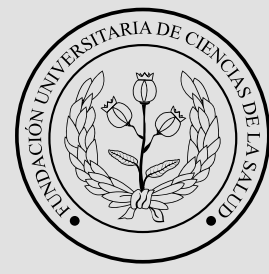

FUCS

\title{
Perspectiva del COVID-19 en Colombia para el año 2021
}

\section{A perspective on the spread of COVID-19 in Colombia by 2021}

${ }^{a}$ Ingeniero. Magister en Gestión de la Tecnología Educativa, Especialista en Administración de la Informática Educativa. Docente de matemáticas e Investigador, Secretaría de Educación de Soacha, Cundinamarca.

\section{R E S U M EN}

Introducción: el síndrome respiratorio agudo severo coronavirus 2 (SARS-CoV-2) se ha dispersado velozmente por todo el mundo, incluida la importancia relativa de varias vías de transmisión, el papel de las infecciones asintomáticas y presintomáticas y la susceptibilidad y transmisibilidad de grupos de edad específicos. Objetivo: presentar una perspectiva del contagio de personas recuperadas y fallecidas por el COVID-19 en Colombia para 2021. Metodología: el método utilizado para calcular el pronóstico fue el modelo ARIMA $(0,1,0)$. Resultados: se estimó que para finalizar el mes de diciembre 2021, se tendrá un número de 4'973.547 personas contagiadas, 4'784.987 personas recuperadas, y 110.159 personas fallecidas. Conclusión: la utilización de modelación matemática ha progresado en grado representativo en las últimas décadas es de gran impulso para ilustrar escenarios eficaces de prevención y control de enfermedades infectocontagiosas, esto con la finalidad de seguir monitoreando el SARS-CoV-2, y poder controlar su velocidad de propagación en Colombia.

Palabras clave: COVID-19, modelación matemática, SARS-CoV-2, pandemia.

(C) 2020 Fundación Universitaria de Ciencias de la Salud - FUCS. Este es un artículo Open Access bajo la licencia CC BY-NC-ND (http://creativecommons.org/licenses/by-nc-nd/4.0/).

Historia del artículo:

Fecha recibido: noviembre 11 de 2020 Fecha aceptado: noviembre 17 de 2020

\section{INFORMACIÓN DEL ARTÍCULO}

\footnotetext{
Autor para correspondencia.

Jorge Enrique Díaz Pinzón jediazp@unal.edu.co
}

DOI

10.31260/RepertMedCir.01217372.1136 
Introduction: severe acute respiratory syndrome coronavirus 2 (SARS-CoV-2) has spread rapidly worldwide, including the relative importance of various transmission routes, the role of asymptomatic and pre-symptomatic infection and the susceptibility and transmissibility among specific age groups. Objective: to present a perspective on the spread of COVID-19 in Colombia by 2021. Methodology: the ARIMA $(0,1,0)$ model was used for predicting future data. Results: the estimated values by the end of December 2021 will be 4'973.547 infections, 4'784.987 recoveries and 110.159 deaths. Conclusion: the use of mathematical modeling have made considerable progress during the last few decades and is a very important tool to illustrate effective scenarios for infectious disease prevention and control, with the aim to further enhance monitoring of the SARS-CoV-2 and controlling its transmission rate in Colombia.

Key words: COVID-19, coronavirus, infectious disease transmission; pandemic.

(C) 2020 Fundación Universitaria de Ciencias de la Salud - FUCS. This is an open access article under the CC BY-NC-ND license (http://creativecommons.org/licenses/by-nc-nd/4.0/).

INTRODUCCIÓN

A comienzos de diciembre 2019 en la ciudad de Wuhan, capital de la provincia de Hubei en China central, se registró un grupo de personas enfermas con un tipo de neumonía desconocida. ${ }^{1}$

El síndrome respiratorio agudo severo coronavirus 2 (SARS-CoV-2) se ha dispersado velozmente por todo el mundo, incluida la importancia relativa de varias vías de transmisión, el papel de las infecciones asintomáticas y presintomáticas y la susceptibilidad y transmisibilidad de grupos de edad específicos. ${ }^{2}$

La enfermedad ocasionada por este nuevo virus se ha designado por consenso internacional COVID-19. El Comité de Emergencias del Reglamento Sanitario Internacional (RSI, 005) manifestó el brote como una emergencia de salud pública de importancia internacional (ESPII) en su reunión del 30 de enero 2020. Posteriormente el 11 de marzo 2020 la OMS lo consideró como una pandemia global. ${ }^{3}$

Según Montesinos citado por Díaz ${ }^{4}$, el empleo de modelos matemáticos para enfermedades infecciosas ha crecido en grado significativo en los años anteriores debido a que proveen información ventajosa para tomar decisiones, y crear medidas activas en el control o erradicación de una enfermedad infecciosa. Estos modelos son muy favorables porque sujetan propiedades esenciales de la dispersión de una enfermedad de una forma sintética.

El pronóstico atañe a la predicción de un evento de interés (EI) después de su inicio. El término hace referencia a posibles resultados de un EI y a la frecuencia con la que se puede esperar que se originen. ${ }^{5}$ Los modelos matemáticos se utilizan para entender las transiciones epidemiológicas críticas y predecir los parámetros. Con frecuencia el ajuste de la curva epidemiológica, los datos de vigilancia durante la transmisión temprana y otros modelos epidemiológicos se han empleado para realizar pronósticos de la pandemia de la COVID-19 en el mundo. ${ }^{6-9}$
El objetivo de este trabajo de investigación es presentar una perspectiva del contagio de personas recuperadas y fallecidas por el COVID-19 en Colombia para 2021; el método utilizado para calcular el pronóstico fue el modelo ARIMA $(0,1,0)$, teniendo en cuenta el registro de la información por parte del Instituto Nacional de Salud ${ }^{10}$, hasta el 6 de noviembre 2020 punto de vista económico, social y de salud.

\section{METODOLOG ÍA}

El trabajo de investigación se realizó mediante un enfoque cuantitativo, es aquella en la que se acumulan y examinan datos cuantitativos sobre variables y estudia las propiedades y fenómenos cuantitativos, según Hurtado y Toro (1998) citado por Díaz. ${ }^{11}$

\section{MODELO ARIMA $(P, D, Q)$}

El modelo ARIMA (Autoregresive Integrated Moving Average), admite referir un valor como una función lineal de datos anteriores y errores convenidos al azar, puede contener un componente cíclico o estacional. ${ }^{12,13}$

Los modelos ARIMA se erigen a partir de los modelos ARMA, pero estimando que la serie en estudio para que sea estacionaria en media tendrá que diferenciarse una serie de veces.

Un modelo ARIMA (p, d, q) es un modelo ARMA (p, q) sobre la serie diferenciada d veces. Es decir, su expresión algebraica, será:

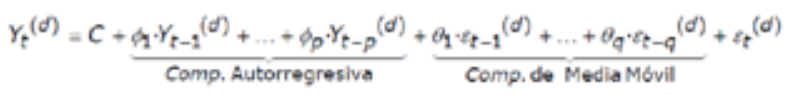


donde $\mathrm{Yt}\left({ }^{\mathrm{d}}\right)$ es la serie de las diferencias de orden, d y $\left.\varepsilon t^{\mathrm{d}}\right)$ es la serie de los errores que se cometen en la serie anterior. 14

Regularmente el orden de diferenciación d, entero, oscila entre 0 y 2. Una vez hemos visto el procedimiento de un modelo ARIMA, podemos afirmar que este se puede precisar como un modelo de regresión lineal múltiple, donde la variable dependiente es la propia serie (diferenciada o no) y las variables independientes son valores de la serie y valores de los errores de ajuste pasados hasta unos órdenes p y q, comparativamente. ${ }^{14}$

\section{POBLACIÓN}

Esta investigación se centró en la población de contagiados, recuperados y personas fallecidas por COVID-19, con la información proveniente del Instituto Nacional de Salud, en el periodo comprendido entre el 6 de marzo al 5 de noviembre 2020. Se utilizó el paquete estadístico SPSS v.25.

\section{RES ULTADOS}

En la tabla 1, se observa la descripción del modelo ARIMA (p, d, q), para los datos acumulados para personas contagiadas, recuperadas y fallecidas por COVID-19.

Tabla 1. Descripción del modelo ARIMA

\begin{tabular}{l|l|ll|}
\hline ID de modelo & Contagiados-Acu & Modelo_1 & ARIMA $(0,1,0)$ \\
\hline ID de modelo & Recuperados-Acu & Modelo_1 & ARIMA $(0,1,0)$ \\
ID de modelo & Fallecidos-Acu & Modelo_1 & ARIMA $(0,1,0)$ \\
\hline
\end{tabular}

En la figura 1 se aprecia la proyección de personas contagiadas para 2021. Al finalizar enero de 2021 se proyecta un número de 1'914.750 personas contagiadas por COVID-19 hasta llegar a finalizar el mes de diciembre con 4'973.547 de personas contagiadas.

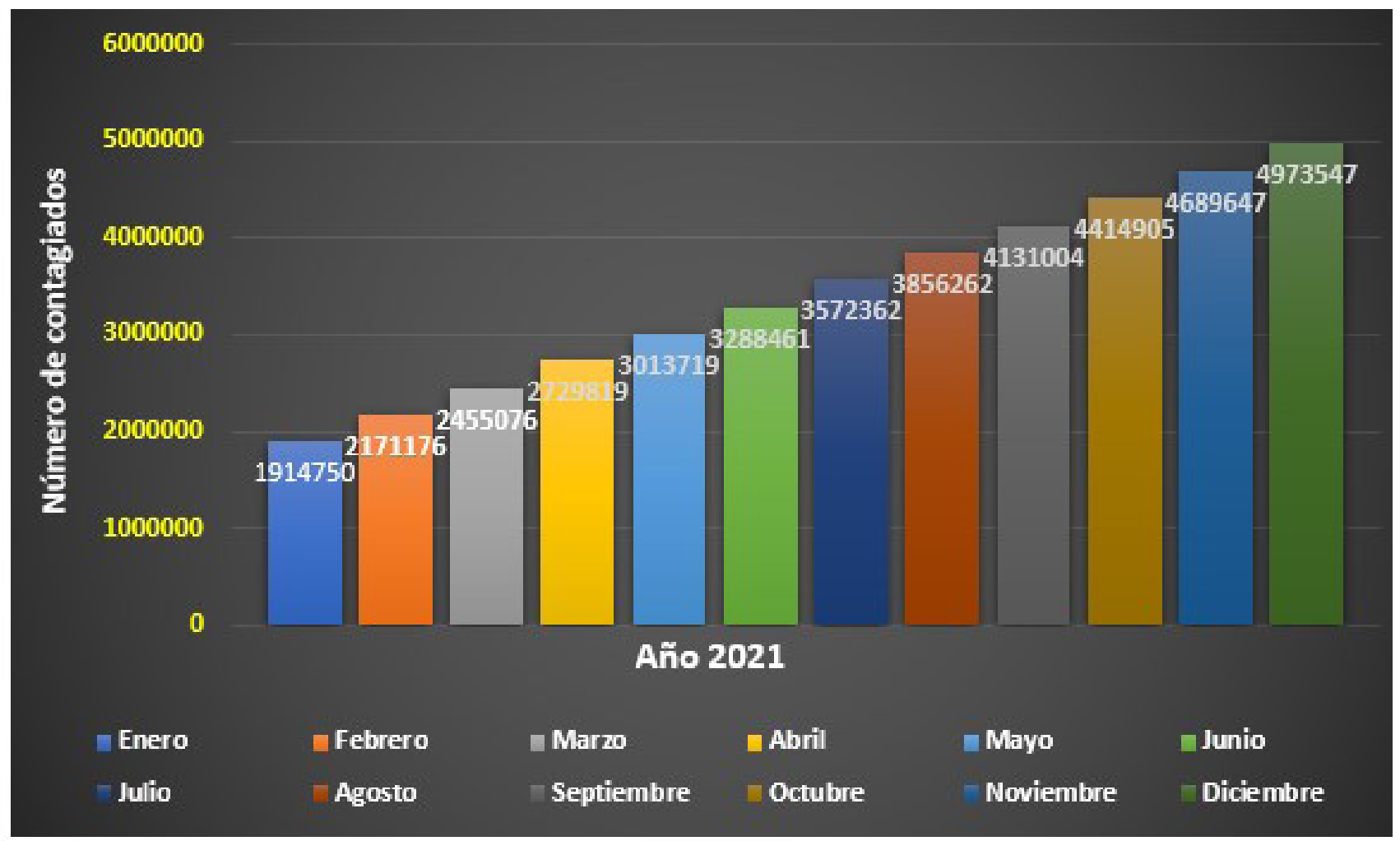

Fuente: el autor.

Figura 1. Número de contagiados por COVID-19 para el año 2021

En la figura 2 se observa la proyección de personas recuperadas para 2021. Al finalizar el mes de enero 2021 se proyecta un número de 1'710.029 personas contagiadas por COVID-19 hasta llegar a finalizar el mes de diciembre con 4'784.987 de personas recuperadas.
En la figura 3 se evidencia la proyección de personas fallecidas para 2021. Al finalizar el mes de enero 2021 se proyecta un número de 48.317 personas fallecidas por COVID-19 hasta llegar a finalizar el mes de diciembre con 110.159 de personas fallecidas. 


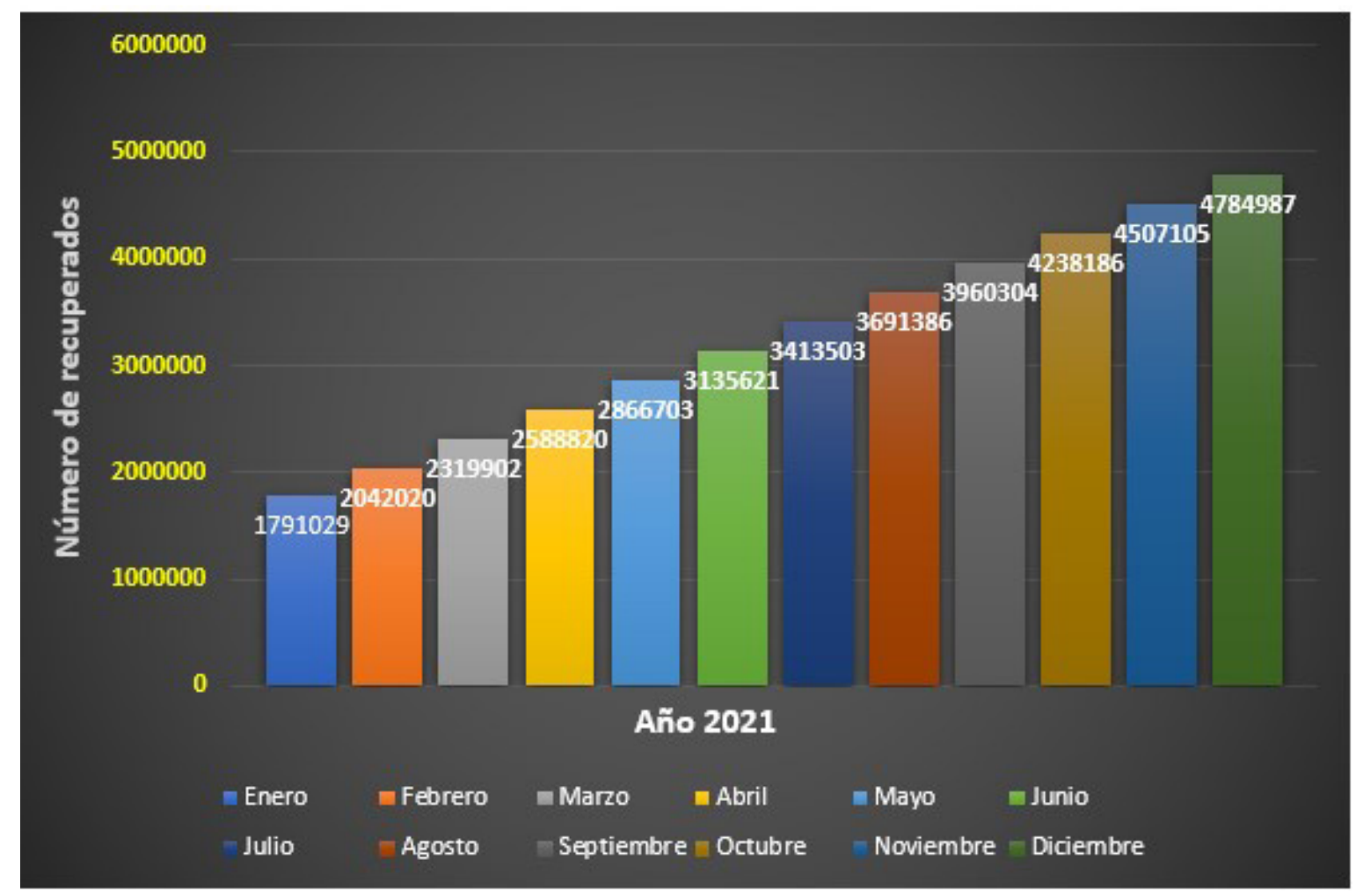

Fuente: el autor.

Figura 2. Número de recuperados por COVID-19 para el año 2021

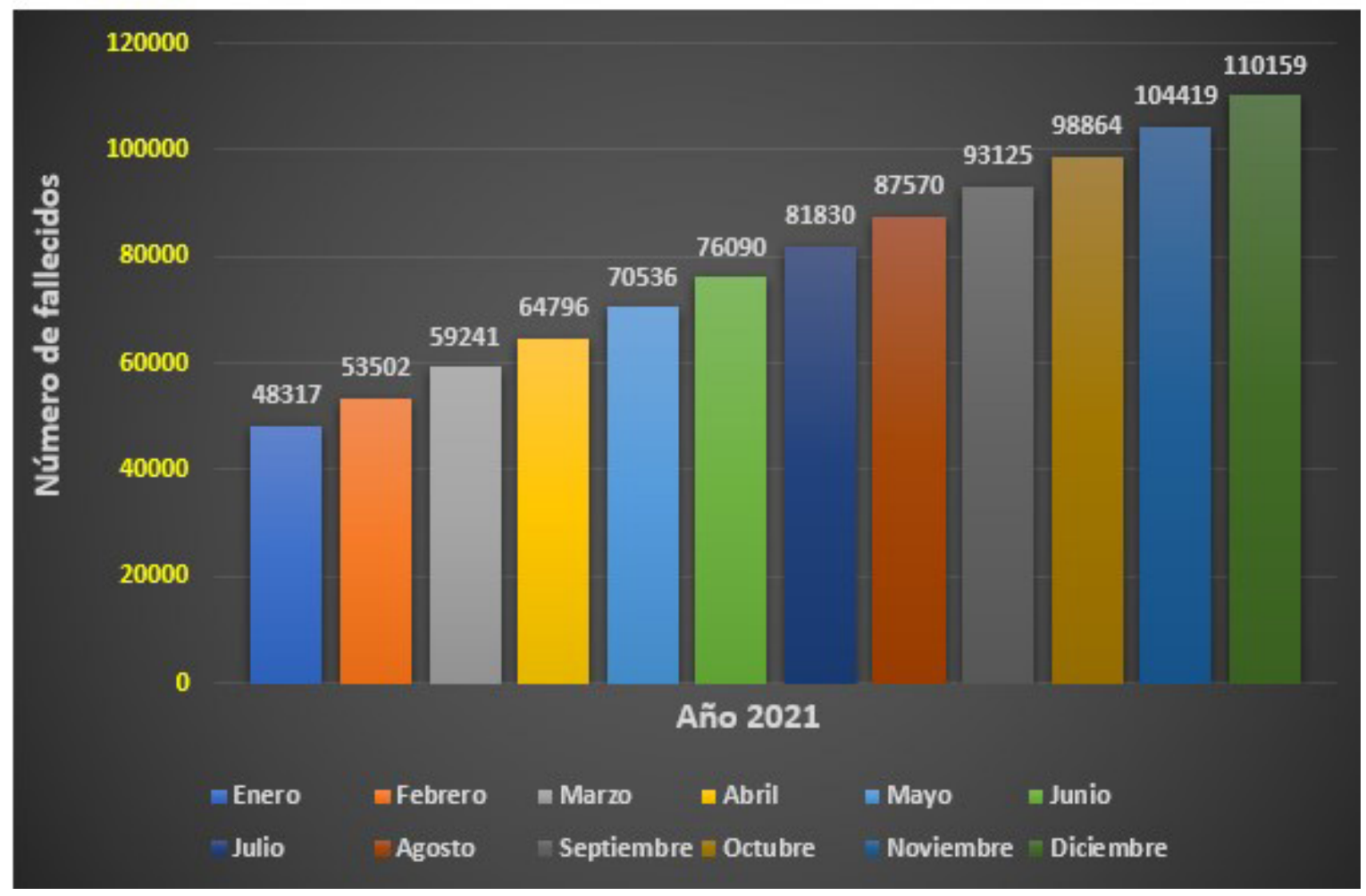

Fuente: el autor.

Figura 3. Número de fallecidos año 2021. 
Hay que analizar el efecto del COVID-19 en otros ámbitos como la economía y la educación, el país enfrenta una difícil situación entre contener la actividad económica o contener una pandemia. Cuanto antes se tomen medidas inflexibles para aplacar la propagación del virus, cuanta más propagación, mayor será el impacto en la economía a corto plazo, pero con las medidas sanitarias y el teletrabajo se puede dominar la pandemia. ${ }^{15}$

Las instituciones deben considerar los comentarios de los estudiantes y los docentes para mejorar sus planes de contingencia y por lo tanto, Hay que analizar el efecto del COVID-19 en otros ámbitos como la economía y la educación, el país enfrenta una difícil situación entre contener la actividad económica o contener una pandemia. Cuanto antes se tomen medidas inflexibles para aplacar la propagación del virus, cuanta más propagación, mayor será el impacto en la economía a corto plazo, pero con las medidas sanitarias y el teletrabajo se puede dominar la pandemia. ${ }^{15}$

Las instituciones deben considerar los comentarios de los estudiantes y los docentes para mejorar sus planes de contingencia y por lo tanto, tener un impacto positivo en la enseñanza. De igual manera se deben realizar procesos de formación permanente en el uso de las tecnologías de la información y la comunicación para estudiantes y docentes, esto para fortalecer este campo en beneficio de la comunidad educativa del país. ${ }^{16}$

\section{CONCLUSIONES}

De acuerdo con la proyección del COVID-19 para 2021 en Colombia por el método ARIMA $(0,1,0)$, se estimó que para finalizar el mes de diciembre se tendrá un número de 4'973.547 personas contagiadas, 4'784.987 personas recuperadas, y 110.159 de personas fallecidas. Ahora, la difusión del SARSCoV-2, la situación nacional e internacional, la carencia por el momento de una vacuna efectiva contra el COVID-19, admite la obligación de continuar con las medidas de aislamiento selectivo en los próximos meses y años, con la finalidad de mitigar la enfermedad en Colombia, y poder de esta forma impedir que se presenten rebrotes en el país. El autor recomienda utilizar otros modelos de pronóstico, por ejemplo, el modelo de suavizado exponencial de Brown según IBM citado por Díaz. ${ }^{17,18}$ Este método es muy análogo a un modelo ARIMA con cero órdenes de autoregresión, dos órdenes de distinción y dos órdenes de media móvil, con el coeficiente para el segundo orden de media móvil igual al cuadrado de la mitad del coeficiente de primer orden. La utilización de modelación matemática ha progresado en grado representativo en las últimas décadas y son de gran impulso para ilustrar escenarios eficaces de prevención y control de enfermedades infectocontagiosas, esto con la finalidad de seguir monitoreando el SARS-CoV-2, y poder controlar su velocidad de propagación en Colombia.
DECLARACIÓN CONFLICTO

El autor declara no tener ningún conflicto de interes.

\section{REFERENCIAS}

1. Li Q, Guan X, Wu P, Wang X, Zhou L, Tong Y, et al. Early Transmission Dynamics in Wuhan, China, of Novel Coronavirus-Infected pneumonia. N Engl J Med. 2020;382(13):1199-1207. Doi:10.1056/NEJMoa2001316

2. Lee CE, Wada N, Grabowski KM, Gurley E, Lessler J. The engines of SARSCoV-2 spread. Doi: 10.1126/science.abd8755

3. ISCIII. Informe sobre la situación de COVID-19 en España. 2020 [Internet]. 2020 [citado 2020 noviembre 03]. Recuperado de: https://www. isciii.es/QueHacemos/Servicios/VigilanciaSaludPublicaRENAVE/ EnfermedadesTransmisibles/Paginas/InformesCOVID-19.aspx

4. Díaz Pinzón, JE. Precisión del pronóstico de la propagación del COVID-19 en Colombia. Repert. Med. Cir. 2020;29(Supl.1):27-33. Doi: 10.31260/ RepertMedCir.01217372.1045

5. Manterola, C; Santander, C; Otzen, T. Cómo valorar e interpretar un artículo sobre pronóstico. Rev Chil Cir. 2013;65(1):77-84. Doi: 10.4067/S071840262013000100015

6. Singh RK, Rani M, Bhagavathula AS, Sah R, Rodriguez-Morales AJ, Kalita $\mathrm{H}$, et al. Prediction of the COVID-19 pandemic for the top 15 affected countries: Advanced Autoregressive Integrated Moving Average (ARIMA)model.JMIRPublic Health Surveill. 2020;6(2):e19115. Doi: 10.2196/19115

7. Kucharski AJ, Russell TW, Diamond C, Liu Y, Edmunds J, Funk S, et al. Early dynamics of transmission and control of COVID-19: a mathematical modelling study. 2020;20(5):553-558. Doi: 10.1016/S14733099(20)30144-4

8. Liu Y, Gayle A, Wilder-Smith A, Rocklöv J. The reproductive number of COVID-19 is higher compared to SARS coronavirus. J Travel Med. 2020;27(2):taaa021. Doi: 10.1093/jtm/taaa021

9. Pirouz B, Shaffiee Haghshenas S, Piro P. Investigating a Serious Challenge in the Sustainable Development Process: Analysis of Confirmed cases of COVID-19 (New Type of Coronavirus) Through a Binary Classification Using Artificial Intelligence and Regression Analysis. Sustainability. 2020;12(6): 2427. Doi: 10.3390/su12062427

10. Instituto Nacional de Salud. Coronavirus (COVID-2019) en Colombia [Internet]. Bogotá: Instituto Nacional de Salud; 2020. [citado 2020 noviembre 7]; Recuperado de: https://www.ins.gov.co/ Noticias/Paginas/Coronavirus.aspx.

11. Díaz-Pinzón, J.E. Correlación y regresión lineal de la evaluación tiempo y puntaje con recurso interactivo flash. INNOVA Research Journal. 2017;2(10):1-8. doi: https://doi.org/10.33890/innova.v2.n10.2017

12. De la fuente S. Modelo ARIMA (p, d, q) [Internet]. 2020 [citado 2020 noviembre 7]; Disponible en: http://www.estadistica.net/ ECONOMETRIA/SERIES-TEMPORALES/modelo-arima.pdf

13. Calvo D. Análisis de series temporales en R. ARIMA. 2018 [Internet]. [citado 2020 noviembre 7]; Disponible en: https://www.diegocalvo. es/analisis-de-series-temporales-en-r-arima/

14. Maté, C. Modelos ARIMA. 2012 [Internet]. [citado 2020 noviembre 7]; Recuperado de: https://es2.slideshare.net/juan_churqui/ modelo-arima-14236175?from_action=save 
15. Quintero Rivera JJ. El Efecto del COVID-19 en la Economía y la Educación: Estrategias para la Educación Virtual de Colombia. Revista Scientific. 2020;5(17):280-291. Doi: 10.29394/Scientific.issn.25422987.2020.5.17.15.280-291

16. Díaz-Pinzón JE. Formación de Docentes en el Uso y Apropiación de las TIC. Innova Research Journal. 2017;2(9): 18-25. Doi: 10.33890/innova. v2.n9.2017.257
17. Díaz-Pinzón JE. Precisión del pronóstico de la propagación del COVID-19 en Colombia. Repert Med Cir. 2020;29(Núm. Supl.1):27-33. Doi: 10.31260/RepertMedCir.01217372.1045

18. Díaz-Pinzón JE. Uso de modelo predictivo para la dinámica de transmisión del COVID-19 en Colombia. Repert Med Cir. 2020;29(Núm. Supl.1):34-44. Doi: 10.31260/ RepertMedCir.01217372.1056 